\title{
Padres y madres homosexuales y bisexuales en Colombia: aproximación a las percepciones sobre la familia
}

\author{
Jair Eduardo Restrepo Pineda* \\ Juliana Jaramillo Jaramillo*
}

\section{Resumen}

Este artículo presenta un análisis a las percepciones que padres y madres homosexuales y bisexuales residentes en Colombia tienen acerca de sus familias. Para tal fin, se realizó una aproximación a los imaginarios y las representaciones sociales que sobre la familia y la orientación sexual poseen estos padres, logrando evidenciar la manera en la cual vinculan ambas categorías para reconfigurar, desde sus experiencias personales, la imagen y la percepción de la familia que han constituido. La metodología empleada fue de carácter cualitativo y tuvo como herramientas de investigación la entrevista semiestructurada y el test de asociación libre de palabras. En total, se entrevistaron 15 padres, de los cuales siete eran lesbianas, cuatro gays y cuatro bisexuales. Se pudo concluir que los padres y madres homosexuales y bisexuales tienen percepciones sobre sus familias en las cuales no se da un rompimiento radical con el modelo de familia tradicional, sino que se presentan continuidades en aspectos tales como la afectividad, el cuidado, los roles de género, los cuales, sin embargo, son valorados y priorizadas de forma distinta por estos padres y madres dentro de las familias que han constituido.

Palabras clave: parentalidad, homosexual, bisexual, progenitores, relaciones familiares.

\footnotetext{
* Corporación Universitaria Minuto de Dios, Bello, Antioquia, Colombia.
} 


\section{Gay and bisexual parents in Colombia: an approach to perceptions of the family}

\section{Abstract}

This article presents an analysis to the perceptions that gay and bisexual fathers and mothers living in Colombia have of their families. For this purpose an approach was made to the imaginaries and social representations of these parents about their family and sexual orientation, so that to demonstrate the way they link both categories to reconfigure, from their personal experiences, the image and perception of the family that they have constituted. The methodology used was of a qualitative, exploratory and descriptive nature, using the semi-structured interview and free word association test as research tools. In total 15 parents were interviewed, of which 7 were lesbians, 4 gays and 4 bisexuals. It was concluded that homosexual and bisexual fathers and mothers have perceptions of their families that suggest no radical break with the traditional family model, but rather shows continuities regarding aspects such as affectivity, care and gender roles, which, however, are valued and prioritized in a different way by these fathers and mothers within the families they have constituted.

Keywords: parenting, homosexual, bisexual, parents, family relationships.

\section{Introducción ${ }^{1}$}

n el contexto colombiano, así como en gran parte de la sociedad
occidental, las representaciones sobre la familia están influenciadas
por la definición clásica de la estructura familiar, según la cual ésta se entiende como una relación monógama entre un varón y una mujer, con una finalidad procreativa y legitimada socialmente a través del matrimonio (Grau, 2016; López, 1998). Sin embargo, las transformaciones que se han producido en la vida familiar dan cuenta de los "diferentes procesos de construcción social de sentido y, por lo tanto, de subjetividades desde las que se construyen distintas formas de vida en familia" (Bárcenas, 2010, p. 78). Así, se observa la existencia de parejas casadas que deciden no

${ }^{1}$ Se agradece el apoyo de CONICYT-Chile, a través del Proyecto Fondecyt no 11160747, en la realización de la investigación, algunos de cuyos resultados se comunican en este artículo. 
tener hijos, la conformación de familias por parejas del mismo sexo, la monoparentalidad por elección y las familias con hijos adoptados. Estos son, entre otros, ejemplos de cambios ocurridos en las familias, que dan cuenta de su diversidad.

En el caso de las familias conformadas por padres y madres homosexuales y bisexuales, hay un doble cuestionamiento implicado, no solo del modelo hegemónico de familia, referido este al

imaginario social [de] un modelo de familia hegemónico idealizado que responde al patriarcado heteronormativo de base cristiana, donde subyace la percepción de que la única familia legítima y completa (y la única que puede llegar a ser feliz) es la conformada por un papá, una mamá, un hijo y una hija (ATSS, 2016, p. 1),

sino también respecto a los prejuicios en torno a las orientaciones sexuales no hegemónicas. Es así como las familias cuyos progenitores tienen una orientación sexual diferente a la heterosexual desafían la norma de la complementariedad de los sexos. Tal situación condujo al desarrollo de investigaciones que se propusieron analizar los efectos que esta nueva forma de estructura familiar tenía sobre los hijos (Weeks et al., 1975; Green, 1978; Golombok et al, 1983). Estos estudios han permitido desvirtuar dos creencias arraigadas: la primera, que los homosexuales no son aptos para ejercer como padres y que, por lo tanto, los hijos tendrán problemas; y la segunda, que la estructura familiar compuesta por padres del mismo sexo no permite un desarrollo psicosocial adecuado y un buen funcionamiento familiar en general. Los investigadores propusieron, a partir de lo anterior, que lo más importante no era la estructura, sino las dinámicas familiares, $y$, en esta medida, han hecho un llamado a ocuparse de los procesos que se viven dentro de estas familias.

Las investigaciones con este enfoque han identificado que, si bien las familias conformadas por lesbianas, gays, bisexuales y personas transgénero (LGBT) no se diferencian radicalmente de las otras, sí existen elementos que las hacen particulares, el principal de ellos es que deben lidiar con 
la estigmatización y la exclusión social a la cual se ven sometidas. Otros elementos que las diferencian son la tendencia a la flexibilidad de los roles, a una crianza menos estereotipada y un mayor énfasis en valores de reconocimiento, tolerancia y respeto por la diversidad.

Diferentes investigaciones se han centrado en analizar la manera como la estigmatización afecta tanto la dinámica familiar como la forma cómo se percibe y vivencia individualmente la paternidad y/o maternidad. Entre estas se encuentran la de Zapata (2013) y la de Vargas et al. (2011). Esta última se ocupó de analizar las experiencias de cuatro hombres y dos mujeres, de orientación homosexual o bisexual, en distintos momentos de sus vidas y bajo roles como el de hijos, parejas y padres, para comprender el papel jugado por su orientación sexual.

En su estudio, Zambrano (2006) busca demostrar cómo el modelo de familia tradicional ha influido en la construcción de la parentalidad por parte de las personas con orientaciones sexuales no heterosexuales y otras identidades de género y sostiene la necesidad de fomentar un cambio social que permita cumplir con las nuevas exigencias y la deconstrucción de las viejas certezas de la antropología, la psicología, el psicoanálisis y la ley, de manera a favorecer la legitimidad de estas nuevas familias dentro de la sociedad. De otra parte, y considerando la diversidad de formas familiares, la Corte Constitucional de Colombia ha propuesto ir más allá de la perspectiva naturalista que establece el origen de la familia en la unión afectiva que se da entre un hombre y una mujer, para adoptar un sentido amplio de familia desde el cual ésta se entiende como

aquella comunidad de personas emparentadas entre sí por vínculos naturales o jurídicos, que funda su existencia en el amor, el respeto y la solidaridad, y que se caracteriza por la unidad de vida o de destino que liga íntimamente a sus integrantes más próximos (Corte Constitucional de Colombia, 2003).

Este concepto es más incluyente y permite evidenciar los múltiples factores que intervienen en la construcción de las representaciones sociales sobre la familia, alejándose de un modelo único e inmutable y reconociendo 
aspectos tales como la diversidad de estructuras, arreglos, formas y roles que se pueden presentar al interior de la familia, sin condicionarlos a determinados sexos u orientaciones sexuales, además de dar prioridad a los vínculos afectivos, de solidaridad y de cuidado que se establecen entre sus miembros.

No obstante, y a pesar de la diversidad de formas familiares y los cambios sociales ocurridos, la heteronormatividad sigue teniendo una fuerte influencia en las personas LGBT, evidenciándose en la investigación que los imaginarios y las representaciones sociales sobre la familia que expresan los individuos entrevistados están influenciados por la heteronormatividad. De esta manera,

las relaciones entre personas del mismo sexo no escapan necesariamente a las presiones sociales de imitar las prevalentes normas del sexo: heterosexual, coital, en relaciones de pareja, monógamas, en casa, no comerciales, por amor, y entre miembros de la misma generación (Rubin et al., 2001, p. 27, citado en Pichardo et al., 2015, p. 197).

Por su parte, Gallego y Giraldo (2016) hacen evidente que en las prácticas homoeróticas de las mujeres existe una relación marcada con la heterosexualidad, además,

los vínculos parentales que generan una relación de pareja del mismo sexo no tienen nominación en el lenguaje del parentesco convencional, su permanencia y vinculación con las redes de familia demanda de sus miembros nombrar lo innombrable y significar tal relación (Gallego, 2011, p. 101),

es decir, la representación social de la familia conformada por padres y madres homosexuales y bisexuales es construida por sus miembros en relación con el modelo tradicional. Weston sostiene que "las familias de elección resultaban atractivas en parte porque reintroducían en la organización social de las lesbianas y los gays la apropiación y el sentido subjetivo de crear una cultura" (Weston, 2003, p. 184) y, por tanto, darían origen a otras formas de concebir a la familia, alejadas de los lazos 
biogenéticos y priorizando los vínculos afectivos y de solidaridad que se establecen entre sus miembros.

Este artículo explora las percepciones que padres y madres homosexuales y bisexuales residentes en Colombia construyen acerca de las familias que han conformado. Para tal fin se realizó un análisis de los imaginarios y las representaciones sociales que estos tienen, y la manera en la cual estas familias desafían, reproducen o reconfiguran los modelos hegemónicos de familia.

\section{Metodología}

Con el fin de analizar las percepciones que padres y madres homosexuales y bisexuales residentes en Colombia tienen acerca de sus familias, se diseñó una investigación de tipo cualitativo, en la cual el análisis de las percepciones se realizó a través del estudio de los imaginarios y las representaciones sociales que sobre la familia y la orientación sexual poseen estos padres.

Atendiendo a la dificultad de acceder a este tipo de familias en Colombia, se definió entrevistar al mayor número posible de ellas que cumpliesen con los siguientes criterios de inclusión:

- residir en los departamentos de Caldas, Quindío, Risaralda o Antioquia;

- los padres debían autoidentificarse como homosexuales o bisexuales;

- $\quad$ tener por lo menos un hijo (a);

- el acceso a la paternidad y/o maternidad se podría haber dado por cualquier medio (adopción, acogida, tratamientos de reproducción asistida, biológico, otros).

Se contactaron en total 15 familias cuyos progenitores se autoidentificaban como homosexuales o bisexuales y residían en los departamentos de Caldas, Quindío, Risaralda (Eje Cafetero) y en el departamento de Antioquia en Colombia. Esta región geográfica fue seleccionada siguiendo la delimitación elaborada por Gutiérrez de Pineda (1975), quien la define como el complejo 
cultural antioqueño o de la montaña, donde las características sociales, económicas y culturales compartidas determinan sus estructuras y dinámicas familiares.

Se utilizaron como herramientas metodológicas la entrevista semiestructurada, en la cual se indagaba por diversos aspectos tanto de la conformación como de las dinámicas familiares. Entre los aspectos consultados están las características sociodemográficas de las familias, las relaciones de parentesco, la afectividad, la sexualidad, el acceso a la paternidad y/o maternidad, las relaciones de género, entre otros.

Además, se utilizó el test de asociación libre de palabras, que permitió indagar sobre la percepción de la realidad por parte de un grupo social a partir de una composición semántica preexistente. Para Bardin (2002), dicha composición es muy precisa e imaginaria, y está estructurada alrededor de elementos simbólicos simples que constituyen y orientan la información objetiva o la percepción real del objeto de estudio.

Esta técnica se desarrolló a través de una serie de términos inductores, a saber: familia, padres, parentesco, sexualidad, género, homosexualidad y bisexualidad, para que los entrevistados verbalizaran palabras relacionadas con estos estímulos, lo que permitió "colocar en evidencia universos semánticos de palabras que agrupan determinadas poblaciones" (Coutinho, 2001, p. 98).

\section{Las familias constituidas por padres y madres homosexuales y bisexuales en Colombia}

Las entrevistas a los padres fueron contestadas por nueve mujeres y seis varones, de los cuales siete son lesbianas, cuatro gays y cuatro bisexuales. La edad promedio de los entrevistados es de 36 años, con una edad mínima de 24 años y una máxima de 59. En cuanto a los estados civiles, dos mujeres se encuentran casadas, una de ellas porque aún no se ha divorciado de su expareja, aunque no convive con ésta, mientras que, en el otro caso, la mujer se encuentra casada con su pareja del mismo sexo, dicho matrimonio se llevó a cabo en el exterior ya que cuando tomaron 
esta decisión en Colombia no se encontraba reconocida legalmente la unión entre dos personas del mismo sexo. Solo una de las entrevistadas informó que se encontraba divorciada, mientras que seis personas informaron que su estado civil era soltero, correspondiendo en todos los casos a varones. Finalmente, seis personas manifiestaron que se encuentran en unión de hecho, las cuales, en su totalidad, corresponden a mujeres (cuadro 1).

\section{Cuadro 1 - Caracterización sociodemográfica de las familias con padres y madres homosexuales y bisexuales en Colombial}

\begin{tabular}{|c|c|c|c|c|c|c|c|c|}
\hline $\begin{array}{l}\text { ID } \\
\text { Familia }\end{array}$ & Sexo & $\begin{array}{l}\text { Orientación } \\
\text { sexual }\end{array}$ & Edad & Estado civil & $\begin{array}{l}\text { Nivel } \\
\text { educativo }\end{array}$ & $\begin{array}{l}\text { Actividad } \\
\text { económica }\end{array}$ & Ingresos & $\begin{array}{l}\text { Tipología } \\
\text { Familiar }\end{array}$ \\
\hline 1 & Mujer & Lesbiana & 36 & Casada & Bachillerato & $\begin{array}{l}\text { Actividades } \\
\text { del hogar }\end{array}$ & $\mathrm{SMLV}^{*}$ & $\begin{array}{l}\text { Familia } \\
\text { nuclear }\end{array}$ \\
\hline 2 & Varón & Bisexual & 29 & Soltero & Pregrado & Estudiante & $\begin{array}{l}<1 \\
\text { SMLV }\end{array}$ & $\begin{array}{l}\text { Familia } \\
\text { extensa }\end{array}$ \\
\hline 3 & Varón & Gay & 40 & Soltero & Pregrado & Empleado & $\begin{array}{l}3 \text { y } 5 \\
\text { SMLV }\end{array}$ & $\begin{array}{l}\text { Familia } \\
\text { extensa }\end{array}$ \\
\hline 4 & Mujer & Lesbiana & 28 & $\begin{array}{l}\text { Unión de } \\
\text { hecho }\end{array}$ & Tecnología & Empleada & 2 SMLV & $\begin{array}{l}\text { Familia } \\
\text { nuclear }\end{array}$ \\
\hline 5 & Mujer & Lesbiana & 59 & $\begin{array}{l}\text { Unión de } \\
\text { hecho }\end{array}$ & Posgrado & Empleada & $\begin{array}{l}3 \text { y } 5 \\
\text { SMLV }\end{array}$ & $\begin{array}{l}\text { Familia } \\
\text { extensa }\end{array}$ \\
\hline 6 & Varón & Bisexual & 24 & Soltero & Técnico & Empleado & $\begin{array}{l}2 \mathrm{y}^{3} \\
\text { SMLV }\end{array}$ & $\begin{array}{l}\text { Familia } \\
\text { extensa }\end{array}$ \\
\hline 7 & Mujer & Bisexual & 24 & $\begin{array}{l}\text { Unión de } \\
\text { hecho }\end{array}$ & Bachillerato & Estudiante & 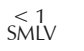 & $\begin{array}{l}\text { Familia } \\
\text { nuclear }\end{array}$ \\
\hline 8 & Mujer & Lesbiana & 31 & $\begin{array}{l}\text { Unión de } \\
\text { hecho }\end{array}$ & Bachillerato & $\begin{array}{l}\text { Trabajo } \\
\text { informal }\end{array}$ & 3 SMLV & $\begin{array}{l}\text { Familia } \\
\text { extensa }\end{array}$ \\
\hline 9 & Mujer & Lesbiana & 20 & $\begin{array}{l}\text { Unión de } \\
\text { hecho }\end{array}$ & Bachillerato & $\begin{array}{l}\text { Trabajo } \\
\text { informal }\end{array}$ & 3 SMLV & $\begin{array}{l}\text { Familia } \\
\text { nuclear }\end{array}$ \\
\hline 10 & Varón & Gay & 39 & Soltero & Posgrado & Empleado & 6 SMLV & $\begin{array}{l}\text { Familia } \\
\text { extensa }\end{array}$ \\
\hline 11 & Varón & Gay & 25 & Soltero & Pregrado & Empleado & $\begin{array}{l}1 \\
\text { SMLV }\end{array}$ & $\begin{array}{l}\text { Familia } \\
\text { reconstituida }\end{array}$ \\
\hline 12 & Varón & Gay & 42 & Soltero & Técnico & Empleado & $\begin{array}{l}1 \mathrm{Y}^{2} \\
\text { SMLV }\end{array}$ & $\begin{array}{l}\text { Familia } \\
\text { extensa }\end{array}$ \\
\hline 13 & Mujer & Bisexual & 47 & Casada & Posgrado & Empleada & 6 SMLV & $\begin{array}{l}\text { Familia } \\
\text { nuclear }\end{array}$ \\
\hline 14 & Mujer & Lesbiana & 37 & $\begin{array}{l}\text { Unión de } \\
\text { hecho }\end{array}$ & Posgrado & Empleada & $\begin{array}{l}3 \mathrm{y}^{5} \\
\text { SMLV }\end{array}$ & $\begin{array}{l}\text { Familia } \\
\text { nuclear }\end{array}$ \\
\hline 15 & Mujer & Lesbiana & 49 & Divorciada & Tecnología & $\begin{array}{l}\text { Trabajo } \\
\text { Independente }\end{array}$ & $\begin{array}{l}1 \\
\text { SMLV }\end{array}$ & $\begin{array}{l}\text { Familia } \\
\text { reconstituida }\end{array}$ \\
\hline
\end{tabular}

Nota ${ }^{*}$ ): El Salario Mínimo Legal Vigente (SMLV) mensual en Colombia es de 877.803 pesos, equivalentes aproximadamente a 267 dólares mensuales.

Fuente: Elaboración propia (2019). 
Por su parte, las formas de organización familiar corresponden mayoritariamente a familias extensas, con siete casos, estando conformadas por parientes pertenecientes a distintas generaciones, los cuales habitan en un mismo hogar. Generalmente se encuentran presentes en este tipo de familias los hijos, los padres y los abuelos. Le siguen las familias nucleares con seis casos, las cuales están formadas por los miembros de un único núcleo familiar, es decir por uno o los dos padres y sus hijos. Finalmente, se encuentran las familias reconstituidas, con dos casos, siendo aquellas en las cuales uno o ambos miembros de la actual pareja tienen uno o varios hijos de uniones anteriores. A estos casos corresponden las familias constituidas por dos mujeres quienes tuvieron sus hijos en relaciones heterosexuales anteriores a la conformación de la familia homoparental.

\section{Acceso a la maternidad y paternidad}

En cuanto al acceso a la maternidad y la paternidad, esta se ha dado por dos vías fundamentales: la primera de ellas, la biológica; y la segunda, la acogida. En el primero de los casos los hijos provienen generalmente de relaciones heterosexuales anteriores a la familia constituida actual. Dicha vía es más frecuente entre las mujeres, ya que, como lo sostiene MartínezConde (2012), en el caso de los varones este tipo de método suele ser muy burocrático, emocionalmente demandante y, en la mayor parte de los casos, no es exitoso, generando frustración en los padres. Además, la vía biológica también se hace evidente en los casos de varones y mujeres bisexuales, quienes tuvieron sus hijos en el marco de relaciones heterosexuales.

Como lo evidencian las entrevistas, una mujer lesbiana puede tener diversas opciones para acceder a la maternidad, una de ellas las relaciones sexuales solamente con la finalidad de quedar embarazada. En palabras de una entrevistada,

cuando quise mi segundo hijo, yo quería una niña. Entonces, simplemente busqué un prospecto y dije, bueno, que mi hija me salga bonita; como quien dice, lo utilicé y adiós, tampoco viví con él. (Salomé, lesbiana, 31 años). 
Sin embargo, esto puede significar un riesgo de adquirir alguna infección de transmisión sexual o de verse involucrada en algún tipo de problema si el hombre elegido es conocido y decide reclamar sus derechos como progenitor (Goldberg, 2010).

Por su parte, los varones homosexuales acceden a la paternidad a través de procesos de acogida, los cuales, según Pichardo (2009), permiten crear un vínculo de parentalidad ya sea por un tiempo limitado o, si la familia del niño o la niña lo permite, permanentemente. Es así como los varones homosexuales, y en algunos casos los bisexuales, entrevistados se han hecho cargo de menores de edad que tienen algún parentesco con estos, generalmente sobrinos.

Yo no lo había pensado nunca, y no me preguntaron "¿usted quiere responsabilizarse?", a mí me dijeron: "usted tiene que hacerse cargo del niño porque pasó esta y esta situación...". Entonces eso fue inmediatamente, pues tomé la decisión de aceptar la custodia del niño, al día siguiente viaje hacia donde él estaba... todos esos trámites se hicieron en Bienestar Familiar, firmé y desde ese día estoy completamente a cargo de él. (Juan, homosexual, 39 años).

A diferencia de las mujeres, los varones no han elegido voluntariamente el acceso a la paternidad, ya que los hijos que tienen bajo su protección han llegado a sus vidas de diversas formas, pero generalmente en momentos de crisis familiar.

Solo en dos de los casos de los varones entrevistados los hijos son biológicos, sin embargo, tampoco responden a un deseo de acceder a la paternidad. En el primer de los casos, corresponde a una situación imprevista al iniciar la vida sexual - en palabras del entrevistado,

no fue una decisión concertada ni participativa el tener una hija. En ese momento creo que estaba muy joven y se dio una relación sexual con una chica a esa edad, de tener relaciones sin protección, entonces no fue una decisión concertada a modo de tener una hija, no. (Sebastián, bisexual, 29 años). 
El segundo caso corresponde a una serie de controles sociales que empujaron al entrevistado a responder según los parámetros heteronormativos exigidos a los varones:

Por el contexto de género, por la imposición de un sistema binario, porque esa fue la idea que me vendieron, de que para realizarme como persona y como hombre biológico que lo mejor para mí era tener una familia, que era tener unos hijos, esa fue la idea que me vendieron y que me tenía que casar. (Norberto, homosexual, 42 años).

Sin embargo, el hecho de que el acceso a la paternidad haya sido una decisión donde influyeron otros factores, independientes a la propia voluntad del entrevistado, no tiene relación alguna con las responsabilidades que tienen estos con sus hijos. La paternidad entonces se convierte, para el varón, en una forma a través de la cual se alcanza el estatus de adulto (Fuller, 2000; 2001; Olavarría, 2001). Además, esta no solo tiene un componente biológico, sino que incluye uno social, ya que, al tiempo de transcender genéticamente, también permite traspasar la herencia social, por tanto, el traspaso de los valores sociales, el parentesco social y la herencia del apellido (Costa, 2002 apud Martínez-Conde, 2012). Así, el componente biológico como el social son fundamentales dentro de la cultura antioqueña y del eje cafetero, elementos que permiten "la existencia de un profundo machismo tropical y una imagen catártica de la fecunda realización, como características propias de las facetas de la personalidad básica masculina" (Gutiérrez de Pineda, 1975, p. 403).

\section{Percepciones sobre la familia constituida}

De manera general, el análisis de los discursos de los entrevistados ha permitido considerar algunos aspectos que determinan la percepción que tienen sobre las familias que han constituido. Dichas percepciones vienen determinadas por las relaciones afectivas y de cuidado que se establecen entre sus miembros y por los roles que asumen cada uno de ellos y que constituyen las dinámicas familiares, los aspectos sociodemográficos y las 
trayectorias de vida familiar de los padres, así como por la heteronormatividad que viene a influir en la forma como se conciben como familia.

La dinámica familiar se puede entender como la manera en que los miembros se relacionan en cuanto a aspectos como el tipo de apego, la comunicación, la normas, los roles, la toma de decisiones y la manera de resolver los conflictos (Hernández, 1997; Quintero, 1997). Es decir, las relaciones que se establecen entre los miembros de la familia van más allá de cómo está conformada esta o la orientación sexual de los padres, siendo primordial que se cumplan las funciones de acogida y protección idóneas que logren crear y consolidar espacios de protección, afecto y estimulación, especialmente para quienes se encuentran en las primeras etapas de desarrollo. Esto vendría a corroborar lo que la mayoría de las investigaciones han demostrado sobre el buen desarrollo familiar, el cual no está determinado por la estructura de la familia, sino por los procesos que se viven dentro de esta (Patterson 2017; 2009; Goldberg et al., 2014; González; Sánchez, 2003). Sin embargo, es necesario señalar que, aunque la orientación sexual de los padres no impide que haya un buen funcionamiento familiar, este aspecto sí trae consigo ciertas particularidades (Stacey; Biblarz, 2001).

En todas las familias las dinámicas familiares constituyen un proceso de negociación, apropiación o ruptura de las relaciones que se establecen entre sus miembros, de tal manera que se pone en funcionamiento una estructura que proporciona sentido y significado a quienes hacen parte de una unidad familiar. Estos procesos se dan en los ámbitos de la autoridad, la toma de decisiones, la proveeduría económica, la afectividad y la comunicación. Las familias conformadas por padres y madres de orientación no heterosexual no son ajenas a estos procesos; por el contrario, los desarrollan de forma más concertada y reflexiva que en otros casos, pues las situaciones a las cuales se enfrentan, como son por ejemplo la discriminación, la exclusión, la homofobia, y el rechazo familiar en algunos casos, así se los exigen (Jaramillo; Restrepo, 2019). 
Por tanto, es a través de las dinámicas y las estructuras familiares que se inicia la modificación de la percepción sobre la familia tradicional, que da origen a una reconfiguración de la familia constituida por estos padres que se ajusta a sus realidades y especificidades.

\section{Entre los vínculos afectivos y de cuidado y los lazos biogenéticos}

Algunos de los padres y las madres homosexuales y bisexuales ponen el acento en los lazos afectivos y de cuidado como fundamentales para concebir la familia. Así, los conceptos como el amor, la unión, la fuerza, el cuidado, entre otros, aparecen centrales dentro de los discursos de los entrevistados. En palabras de una madre,

porque familia no es sangre, familia no son los hijos, familia es la gente con la que uno escoge estar. Yo creo que a eso de los vínculos de sangre se les da demasiada importancia, cuando familia es de verdad el que está dispuesto a estar ahí y a cuidar (Magdalena, bisexual, 47 años).

De igual manera, al aplicar el test de asociación libre de palabras, se obtuvo una serie de evocaciones referidas a los sentimientos que mantienen y cohesionan las dinámicas familiares, entre ellas se pueden mencionar amor, respeto, afecto, alegría, comunicación, apoyo, cuidado. Además, se incluyen las evocaciones referidas a la pareja y las mascotas.

De manera general, los padres entrevistados hacen énfasis en los aspectos referidos a los lazos afectivos y de cuidado. Esto explicaría por qué, en algunos casos, los hijos que hacen parte de la familia han Ilegado a ésta a través de la acogida, situación que cuestiona aún más el modelo que privilegia los lazos biogenéticos del parentesco tradicional, ya que

[e]l hijo o la hija no nace de ninguna de las personas adultas que lo/a crían, ni tiene vínculos jurídicos con ellos. Sin embargo, los padres de acogida y los/ as hijos/as acogidos/as construyen a menudo vínculos afectivos semejantes a los que construyen padres e hijos/as reconocidos jurídicamente (Mujika, 2005, p. 5). 
En palabras de Grau (2016, p. 31), "la solidaridad que encarga el vínculo familiar trasciende el ámbito estricto del hogar y se extiende a la realidad física de los parientes y de los vínculos emocionales que les mantienen unidos". Es así como, en todos los casos, los hijos en acogida son niños o niñas a los cuales sus padres biológicos no pueden asegurarles el bienestar emocional o los medios materiales para su subsistencia. El testimonio de Julián, un joven bisexual que acogió a un sobrino, así lo corrobora:

resulta que empaticé mucho con los problemas que él tenía, por la falta de amor, por la ausencia de escucha... luego prácticamente se fue para la casa tirado por parte de la familia, no se lo soportaban porque era un niño complicado... entonces así fue que llegó de una forma un poco inesperada porque yo quería que él estuviera en la casa para poder darle una vida digna (Julián, bisexual, 24 años).

El hecho de que los entrevistados privilegien los lazos afectivos y de cuidado sobre los lazos biogenéticos constituye un primer cambio en las representaciones sociales sobre la familia, ya que estas cumplen una función de reconocimiento, pues las representaciones permiten a los actores sociales comprender y explicar sus realidades de modo asimilable y comprensible para ellos (Perera, 2003, p. 18). De este modo, los padres y madres homosexuales y bisexuales instituyen sus familias desde los vínculos afectivos, de cuidado y de solidaridad desplazando la importancia que se le asigna a los lazos biogenéticos como forma privilegiada de constituir familia.

Los aspectos referidos al vínculo afectivo y de cuidado que se establecen entre los miembros de la familia permiten a los padres y madres con orientaciones sexuales diversas exigir sus derechos como cualquier otra persona, entre ellos el derecho a formar una familia y ser reconocidos como tal. Es así como un entrevistado reconoce que la familia trasciende las orientaciones sexuales de los padres y se establece en torno a los vínculos de cuidado y solidaridad entre sus miembros:

Yo creo que sí manejamos un grado de responsabilidad, la persona es consciente independientemente de la orientación sexual que tenga, que 
tiene una responsabilidad no solamente para responder por decir soy papá o soy mamá ya sea lesbiana o gay, yo creo que uno responde por los estudios, por la comida, por el afecto, por el respeto, yo creo que están en todo el derecho (Cesar, homosexual, 42 años).

De este modo, las representaciones sociales cambian y se modifican de manera tal que la familia ya no está determinada por la orientación sexual de los padres o por la forma en la cual acceden a la paternidad o maternidad, sino por los vínculos de solidaridad, afecto y cuidado que se establecen entre sus miembros.

En cuanto a la influencia de los vínculos biogenéticos, estos siguen presentes en las familias constituidas por padres y madres homosexuales o bisexuales, ya que tanto en el test de asociación libre de palabras como en las narraciones de los entrevistados estos se hicieron evidentes, adquiriendo un valor como medios que aseguran el parentesco y lo legitiman socialmente. Así, en el test de asociación libre de palabras se recogieron todas aquellas evocaciones que se refieren a los lazos genéticos que establecen el parentesco consanguíneo, tales como padre, madre e hijos.

Las referencias de los padres y madres entrevistados a los vínculos afectivos y de cuidado, así como a los lazos biogenéticos vendrían a corroborar lo que sostiene Pichardo:

se remite al carácter social del parentesco sin perder de vista la referencia biológica, ya que ésta no desaparece completamente de las concepciones de la familia entre las personas homosexuales, que no escapan a la influencia de las cosmovisiones hegemónicas (Pichardo, 2009, p. 151).

En palabras de un padre,

me gustaría tener una pareja y con muchos hijos, me gustaría tener una familia grande, ya sea con un hombre o con una mujer, pero definitivamente me gustaría tener un hijo con mi propio ADN, sería lindo (Julián, bisexual, 24 años).

En este sentido, dentro del colectivo LGBTI, el lazo biogenético sigue vigente como base del modelo de familia nuclear donde los vínculos 
familiares tienen una importancia fundamental en la vida personal, social y comunitaria. Estos vínculos se pueden considerar como parte del imaginario instituido dentro de la sociedad para concebir la familia.

Finalmente, es necesario mencionar que, en las representaciones sociales sobre la familia, los aspectos biológicos y los elementos afectivos y emocionales se complementan, dándole un nuevo sentido a la familia que los entrevistados han constituido.

\section{Los roles de género dentro de las familias de padres y madres homosexuales o bisexuales son flexibles}

En las familias constituidas por padres y madres homosexuales o bisexuales se presenta una mayor flexibilización en cuanto a los roles de género, ya que estos no existen de forma preestablecida con relación a los sexos de los miembros de la familia. Sin embargo, cuando lesbianas y gays acceden a la maternidad y la paternidad, lo hacen en un contexto heteronormativo, donde los roles se encuentran bien diferenciados por géneros.

Esta situación se origina en lo que Castoriadis (1997) ha denominado institución, es decir las normas, los valores, el lenguaje, las herramientas, los procedimientos y los métodos que permiten hacer frente a las cosas y condicionan la forma de hacer estas. Sin embargo, "tanto las instituciones como sus 'mecanismos' de continuidad se incorporan en el sujeto mediante la producción de subjetividades" (Erreguerena, 2002, p. 41). Ahora bien, dichas subjetividades permiten comprender y reinterpretar la sociedad de modo tal que pueda ser transformada, siendo este el proceso que se lleva dentro de las familias estudiadas, donde los roles de género se redefinen en función de los intereses, las motivaciones y las expectativas personales de sus miembros, rompiendo con los modelos hegemónicos. El siguiente testimonio así lo reafirma: 
sí, todo es parejo, o sea si alguien tiene que arreglar la casa, todos ayudan a arreglar la casa, todos ayudan a hacer algo, independientemente de sus responsabilidades, todos somos iguales (Juan, bisexual, 29 años).

Por tanto, las familias conformadas por lesbianas, gays y bisexuales tienen una mayor disposición a desafiar los estereotipos de género, asumiendo, redefiniendo o reasignando ciertos roles a diferentes miembros de la familia, sin importar su sexo, especialmente aquellos referidos a las actividades vinculadas al cuidado y el hogar. Estos desafíos tienen lugar al interior de la familia a través de una negociación permanente entre sus miembros y, por tanto, Ilevan a una redefinición de sus dinámicas familiares (Blumstein; Schwartz, 1983, apud Carrington, 1999). Es pues a través de las dinámicas familiares que se cristalizan esos imaginarios sociales y se convierten en representaciones sociales, los cuales "circulan, se cruzan y se cristalizan sin cesar a través de la palabra, un gesto, un reencuentro, en nuestro universo cotidiano" (Moscovici, 1979, p. 39).

Ahora bien, los cambios en los roles dentro de las familias estudiadas no se deben única y exclusivamente a la orientación sexual de los padres o de las madres, ya que pueden existir diferentes factores que promuevan dicha flexibilidad. Por ejemplo, situaciones referidas a la entrada o salida de alguna persona a la unidad familiar, las exigencias de los controles sociales, entre otras. Por tanto, en algunos casos, antes de conformarse las familias por parte de homosexuales o bisexuales ya se habían producido modificaciones en los roles de género. Por ejemplo, aquellos referidos a los cambios en la figura de autoridad y la proveeduría económica, cuando, en las trayectorias familiares de algunas de las mujeres entrevistadas, estas se separaron de sus parejas masculinas para conformar una familia monoparental y posteriormente dar origen a una nueva familia. Se puede decir entonces que cualquier transformación en la configuración familiar da lugar a la modificación de las representaciones sociales y que esto no depende exclusivamente de la orientación sexual de los miembros del hogar. 
Dentro de las familias cuyos padres o madres tienen orientaciones sexuales no heterosexuales se presenta una serie de cambios que flexibilizan los roles de género vinculados directamente con la orientación sexual de los padres. El primero de ellos se refiere a que los padres con orientaciones sexuales no hegemónicas no necesariamente preestablecen roles de género dentro de las familias que conforman. Por tanto, los hijos que son criados en las familias diversas posiblemente construirán roles de género más flexibles y equitativos que aquellos hijos de familias donde se presenta una clara división por sexos de las tareas domésticas y la división sexual del trabajo.

Sin embargo, en algunos casos, la presión ejercida por los modelos hegemónicos de familia y masculinidad también se ven reflejados en algunas de las familias estudiadas, las cuales no escapan a este tipo de presiones. Retomando el relato de Arturo:

¿A mi hijo cómo le demuestro mi afecto? soy muy consentidor, le hablo muy tierno, nos abrazamos mucho, lo trato de "nené" todavía, aunque ya está muy grande, pero todavía es "nené", y todos los días jugamos, le hago cosquillas, lo llevamos a la piscina, jugamos lucha, a tirarnos el uno al otro a la piscina, o sea actividades muy masculinas, toca estimularle eso también (Arturo, homosexual, 39 años).

Es así como, dentro de los imaginarios sociales sobre la masculinidad, se vincula esta con la fuerza y el cuerpo, por tanto, algunos de los padres continúan gestionando la tensión que existe entre los modelos de masculinidad dominantes y aquellas percepciones más incluyentes y menos restrictivas que empiezan a construirse en sus propios hogares.

La división de las tareas domésticas está determinada por diferentes factores, entre ellos el acceso a los recursos económicos o el gusto por determinada actividad. En el primero de los casos se observa que quien tiene menores ingresos económicos en relación con su pareja compensaría esta desigualdad asumiendo mayores cargas en las actividades domésticas. Mientras que, por otro lado, existen algunos individuos que tienen mayor 
afinidad o gusto por determinadas tareas, por tal razón eligen ocuparse del hogar o criar a los hijos.

En algunos casos, especialmente en las familias extensas, las tareas domésticas son delegadas a las mujeres que hacen parte del hogar, generalmente las abuelas. Lewin (2009) sustenta que las prácticas relacionadas con la crianza, tales como preparar los alimentos, cuidar a los hijos e hijas, la limpieza del hogar, lavar la ropa, entre otras, están determinadas por el género y, aunque dichas actividades podrían ser desarrolladas por cualquier persona, estas son frecuentemente asociadas con la mujer. Sin embargo, esta situación puede deberse a una forma de apoyo de la familia extensa para el funcionamiento adecuado de las familias entrevistadas, y además supone un aporte por parte de los abuelos al sostenimiento familiar. Algunos entrevistados corroboran esto:

la mamá y la abuela... pero es mi madre la que está al cuidado de ella, o la madre cuando se va para la casa de ella (Juan, bisexual, 29 años).

Si bien en estos casos existe un apoyo por parte de otros miembros de la familia, de manera general son las mujeres las que deben asumir la mayor parte de las tareas del hogar. En el caso de las familias con ingresos económicos altos, las actividades domésticas son encomendadas a otras mujeres, el siguiente testimonio lo confirma:

pues, primero está Dora, Dora llega aquí a las $8: 30$ y se va a las 5:30, entonces si una de las dos va a llegar tarde o no hay nadie con los niños, ahí está Dora y estamos por fuera o lo que sea y nos vamos a demorar un poquito. Dora está ahí (Magdalena, bisexual, 47 años).

Se puede decir que, aunque las mujeres son participes en las labores del hogar, existe una alta implicación por parte de los hijos y las hijas en las tareas domésticas, en la medida de sus capacidades. Además, se evidencia un proceso permanente de educación y sensibilización frente al respeto a la diferencia: 
mi hijo se encarga, por ejemplo, de la cocina, al medio día él sabe que él tiene que arreglar la cocina, él sabe que tiene que arreglar su cuarto, sabe que tiene que doblar su ropa y mantener su cuarto completamente organizado (Luciana, lesbiana, 37 años).

Autores como González et al. (2002) apuntan a la misma conclusión, ya que en sus investigaciones afirman que existe una mayor flexibilidad en los roles de género por parte de los hijos que son criados en familias homoparentales frente a aquellos que provienen de familias heterosexuales. Así mismo sostienen que en "otras investigaciones se han obtenido resultados similares, sobre todo, en lo relativo al desarrollo de roles de género menos tipificados, más flexibles" (Green et al., 1986; Steckel, 1987 apud González et al., 2002, p. 575; Carrington, 1999).

De manera general, se puede decir que, de las 15 familias que participaron en la investigación, nueve presentan una flexibilización con respecto a los roles de género y, de estas, la mayor parte corresponde a tipologías familiares nuclear y extensa. Otros tres hogares fusionan elementos hegemónicos de los roles de género con aspectos de flexibilización de estos, especialmente en lo referido al cuidado y la afectividad; mientras que las restantes tres familias, mantienen una división de roles ajustada a un modelo más hegemónico, en la que se evidencian claramente las funciones que asumen cada uno de los miembros del hogar vinculadas estrechamente con la proveeduría económica o el cuidado y la crianza.

Estas situaciones ponen en evidencia nuevas percepciones de las familias por parte de los entrevistados, donde se construyen roles de género flexibles y en las cuales se trata de construir una división del trabajo que no intenta replicar ni perpetuar el orden de género heterosexual (Lewin, 2009; Pichardo, 2009). Sin embargo, se encuentran inmersas en un contexto social heteronormativo que condiciona estas nuevas formas de organización familiar, las cuales deben responder a las exigencias culturales, sociales y legales de ese contexto (Goldberg, 2010). No obstante, es indudable la importancia que adquieren las familias con padres y madres homosexuales o bisexuales para la flexibilización de los roles de género y para construir relaciones más incluyentes y respetuosas que puedan trascender socialmente. 


\section{Las familias de padres y madres homosexuales o bisexuales son un espacio para ejercer la maternidad y/o paternidad}

Las familias estudiadas rompen uno de los imaginarios sociales más ampliamente consolidados: el de considerar que las personas con orientaciones sexuales no hegemónicas no pueden ejercer la maternidad o la paternidad, ya sea por razones biológicas - al estar compuestas por dos personas del mismo sexo se suponen relaciones estériles - o por motivos psicológicos, al considerarse que la orientación sexual de los padres puede afectar el desarrollo de los hijos, entre otras. Sin embargo, se evidenció que en algunas familias conformadas por gays, lesbianas o bisexuales la decisión de acceder a la maternidad y/o paternidad es mucho más reflexiva que en otros casos, ya que no se encuentra vinculada a las relaciones sexuales exclusivamente y supone una toma de decisiones continúas. Por ejemplo, si el hijo será biológico o adoptado y, si es biológico, decidirse por un tratamiento de reproducción asistida, en el caso de una pareja de mujeres decidir quién asumirá el proceso de gestación, entre otras. Este tipo de decisiones conlleva una alta implicación parental. Por ejemplo, en el caso de Sofía, una mujer bisexual, la cual tuvo que pasar por un tratamiento de fertilidad para acceder a la maternidad:

de hecho, es planeado, yo me hice un tratamiento de fertilidad, porque yo naturalmente con mis órganos sexuales no puedo, pues según el médico me hacen falta hormonas o algo así, entonces me tuvieron que dar unas hormonas y un poco de cosas así, porque los óvulos no eran fértiles, entonces tocó pagar y todo... mi hijo es planeado y para mí eso es un orgullo grande (Sofía, bisexual, 25 años).

Por otra parte, el ejercicio de la paternidad y/o maternidad en las familias analizadas reconfigura el modelo tradicional de familia, al cuestionar los lazos sanguíneos como única forma de parentalidad y al romper la idea de la unión heterosexual como única forma para procrear. Es así como la mayor parte de los varones homosexuales entrevistados tiene hijos en acogida, los cuales, según Pichardo (2009), permiten crear un vínculo de 
parentalidad ya sea por un tiempo limitado o, si la familia del niño o la niña lo permite, permanentemente,

porque la mamá se fue para el exterior hace muchos años y los dejó al cuidado de nosotros, fuimos los cuidadores tanto de la niña, como del niño, entonces pienso que es como una adaptación familiar (Jorge, homosexual, 40 años).

Dentro de las representaciones sociales de las familias estudiadas se justifica la paternidad o la maternidad a través de los vínculos afectivos y de cuidado hacia el hijo y las relaciones que se establecen entre todos sus miembros. Sin embargo, esta no está condicionada por una relación de pareja, tal como se evidencia en las entrevistas, ya que algunos de los padres o madres carecen de esta y aun así acceden a la parentalidad. En este sentido,

[l]a realidad expone que tener una relación afectivo sexual, no es condición sine qua non para acceder y/o mantener la parentalidad, aunque pueda diferenciarse entre aquellas personas que deciden ser madres o padres en solitario - sin tener la necesidad de una pareja para tal proyecto - y aquellas a las que esa soledad les sobreviene (Pichardo et al., 2015, p. 200).

\section{Conclusiones}

La percepción de los padres y madres de las familias que han constituido está condicionada por los lazos afectivos y de cuidado que se establecen entre sus miembros. Sin embargo, se mantienen presentes los aspectos relacionados con los vínculos sanguíneos que podrían considerarse una herencia de los modelos familiares en los cuales estas personas fueron educados, lo que origina que, para algunos de los entrevistados, sea fundamental que sus hijos tengan un vínculo biogenético con ellos.

Por otra parte, se percibe la familia como un espacio propicio y óptimo para la maternidad y la paternidad, rompiendo con aquellos imaginarios que consideraban este tipo de relaciones estériles e inadecuadas para el desarrollo psicológico de niños y niñas. Se observa una alta implicación 
parental y la búsqueda constante del bienestar de los hijos. Además, las experiencias personales - por ejemplo, provenir de una familia extensa generan percepciones diferentes sobre la familia constituida. En el ejemplo, los individuos tienen mayor aceptación con relación al ingreso de otras personas como parte de la familia.

En las familias estudiadas no se da un rompimiento radical entre las representaciones sociales de la familia de origen y de la familia constituida, sino que se presentan continuidades, puesto que la primera se convierte en referente de las personas diversas por orientación sexual. Por ejemplo, aunque en las familias con padres y madres gays, lesbianas o bisexuales se desplaza el lazo biogenético a un segundo orden, este sigue manteniéndose como un referente para entender las estructuras y las dinámicas familiares. Todo esto sucede en un proceso continuo entre lo instituyente y lo instituido, que permite la reconfiguración de la realidad desde las subjetividades de los individuos.

Los cambios sociales, culturales y jurídicos que se están produciendo en Colombia han contribuido a promover la individualización y la autonomía, al igual que la transformación en los modelos de sexualidad, los cuales deben permitir que la sociedad en general acepte y reconozca la diversidad familiar de manera tal que se respete su autonomía y la flexibilidad de sus miembros en la toma de decisiones. Es decir, que los procesos de conformación de las familias homoparentales que se dan en el ámbito privado deben trascender al debate público con argumentos científicos que permitan que las percepciones, los imaginarios y los discursos se vean reflejados en las prácticas sociales.

Frente a los roles de género, se observa que estos son más flexibles y menos tipificados que los observados en las familias heterosexuales, ya que los padres y madres no tienen roles preestablecidos al constituir sus familias, sino que estos se van negociando en el transcurso de la vida familiar y responden más a una cuestión de deseos, motivaciones, necesidades y expectativas que a una cuestión de división sexual del trabajo. 
Abordar el tema de las percepciones sobre la familia que tienen los padres y las madres homosexuales y bisexuales permite entender cómo se establecen las interacciones sociales y evidenciar de qué manera estas transcienden del discurso a la práctica, teniendo en cuenta que este tipo de familias se enfrentan a una serie de prejuicios sociales, de actos de discriminación y de invisibilización social. Por consiguiente, es "fundamental conocer, interpretar y cuestionar el núcleo figurativo de las representaciones sociales alrededor de las cuales se articulan creencias ideologizadas, pues ello constituye un paso significativo para la modificación de una representación y por ende de una práctica social" (Araya, 2002, p. 12). Es decir, la modificación de la representación, así como el cambio en la práctica social son fundamentales en los procesos de reconocimiento y de respeto por la diversidad afectivo-sexual y de género que permitan la inclusión y la normalización de la diversidad familiar por estos factores.

Jair Eduardo Restrepo Pineda es Doctor en Análisis y Evaluación de Procesos Políticos y Sociales, docente e investigador del Programa de Trabajo Social y líder del Semillero de Investigación en Familia, Género y Diversidad de la Facultad de Ciencias Humanas y Sociales de la Corporación Universitaria Minuto de Dios UNIMINUTO, Sede Bello, Antioquia, Colombia

$\equiv$ jair.restrepo@uniminuto.edu

Juliana Jaramillo-Jaramillo es doctoranda en Estudios Interdisciplinarios sobre Desarrollo en la Universidad de los Andes, Bogotá, Colombia.

$\risingdotseq$ j.jaramilloj@uniandes.edu.co

\section{Referencias}

1. ARAYA, Sandra. Las representaciones sociales: ejes teóricos para su discusión. Serie Cuaderno de Ciencias Sociales 127. San Jose: Facultad Latinoamericana de Ciencias Sociales (FLACSO), 2002.

2. ATSS - Asociación de Trabajadores de la Seguridad Social. Modelos de familia. Historia, estado, sociedad. Sitio ATSS, 13 octubre, 2016. Disponible en: http:// www.atss.org.uy/genero-y-discapacidad/modelos-de-familia/ 
3. BÁRCENAS, Karina. Familias diversas: de la institución al movimiento. Estructuras y dinámicas en la reconfiguración del orden. Tesis [Maestría en Comunicación de la Ciencia y la Cultura], 2010. Instituto Tecnológico y de Estudios Superiores de Occidente, Jalisco, 2010.

4. BARDIN, Laurence. Análisis del contenido. Madrid: Editorial Akal Universitaria, 2002.

5. CARRINGTON, Christopher. No place like home. Relationships and family life among lesbians and gay men. Chicago: The University of Chicago Press, 1999.

6. CASTORIADIS, Cornelius. El imaginario social instituyente, Revista Zona Erógena, n. 35, p. 1-9, 1997.

7. CORTE CONSTITUCIONAL DE COLOMBIA. Sentencia C-271 de 1o de abril de 2003, Demanda de inconstitucionalidad contra el numeral $8^{\circ}$ del artículo 140 del Código Civil, 2003.

8. COUTINHO, Maria da Penha. Depressão infantil: uma abordagem psicossocial. João Pessoa: Editora Universitária, 2001.

9. ERREGUERENA, Albaitero M. Corneluis Castoriadis: sus conceptos, Anuario de investigación, n. 2, p. 39-47, 2002.

10. FULLER, Norma. Paternidades en América Latina. Lima: Fondo Editorial de la Pontifica Universidad Católica del Perú, 2000.

11. FULLER, Norma. Masculinidades, cambios y permanencias. Lima: Fondo Editorial de la Pontifica Universidad Católica del Perú, 2001.

12. GALLEGO, Gabriel. Explicación sociodemográfica de la duración de las relaciones de pareja masculina en la Ciudad de México. Papeles de Población, v. 17, n. 67, p. 91-109, 2011.

13. GALLEGO, Gabriel; GIRALDO, Sebastián. Iniciación sexual en mujeres con prácticas homoeróticas en el eje cafetero colombiano. Sexualidad, Salud y Sociedad - Revista Latinoamericana, n. 22, p. 56-72, 2016. https://doi. org/10.1590/1984-6487.sess.2016.22.03.a

14. GOLDBERG, Abbie; GARTRELL, Nanette; GATES, Gary. Research report on LGB-parent families. The Williams Institute. Los Angeles, CA: UCLA School of Law, 2014.

15. GOLDBERG, Abbie. Lesbian and gay parents and their children. Research on the family life cycle. Washington, DC: American Psychological Association, 2010.

16. GOLOMBOK, Susan; SPENCER, Ann; RUTTER, Michael. Children in lesbian and single parent households: psychosexual and psychiatric appraisal. Child Psychology and Psychiatry, n. 24, p. 551- 572, 1983.

17. GONZÁLEZ, María del Mar; SÁNCHEZ, María Ángeles. Las familias homoparentales y sus redes de apoyo social, Revista Portularia, n. 3, p. 207-220, 2003. 
18. GONZÁLEZ, María del Mar; SÁNCHEZ, María Ángeles; CHACÓN, Fernando. El desarrollo infantil y adolescente en familias homoparentales. Informe preliminar. Departamento de Piscología Evolutiva y de la Educación, Universidad de Sevilla, Sevilla, 2002.

19. GRAU, Jorge. Nuevas formas de familia, ámbitos emergentes. Barcelona: Ediciones Bellaterra, 2016.

20. GREEN, Richard. Sexual identity of 37 children raised by homosexual or transsexual parents. American Journal of Psychiatry, n. 135, p. 692-697, 1978.

21. GUTIÉRREZ DE PINEDA, Virginia. Familia y cultura en Colombia. Medellín: Editorial Universidad de Antioquia, 1975.

22. HERNÁNDEZ, Ángela. Familia, ciclo vital y psicoterapia sistémica breve. Bogotá: Editorial El Búho, 1997.

23. JARAMILLO, Juliana; RESTREPO, Jair Eduardo. Padres y madres homosexuales y bisexuales en Colombia. Experiencias de discriminación y estrategias de afrontamiento. Revista Española de Sociologia, v. 28, n. 1, p. 95-112, 2019. https://doi.org/10.22325/fes/res.2018.62

24. LEWIN, Ellen. Gay fatherhood. Narratives of family and citizenship in America. Chicago: The University of Chicago Press, 2009. https://doi.org/10.7208/ chicago/9780226476599.001.0001

25. LÓPEZ, María Victoria. Familia, sexo y género en la España moderna. Studia Histórica: Historia Moderna, n. 18, p. 105-135, 1998.

26. MARTÍNEZ-CONDE, Macarena. ¿Nuevas familias? Explorando el potencial distintivo de construcciones discursivas en situaciones de homoparentalidad. Departamento de psicología social, Universidad Autónoma de Barcelona, 2012.

27. MOSCOVICI, Serge. El psicoanálisis, su imagen y su público. Buenos Aires, Argentina: Huemul, 1979.

28. MUJIKA, Inmaculada. Modelos familiares y cambios sociales: la homoparentalidad a debate. ALDARTE: Centro de Atención a gays, lesbianas y transexuales, Bilbao, 2005.

29. OLAVARRÍA, José. Y todos quieren ser (buenos) padres. Varones de Santiago de Chile en conflicto. Santiago: FLACSO 2001.

30. PATTERSON, Charlotte. Parents' sexual orientation and children's development, Child Development Perspectives, v. 11, n. 1, p. 45-49, 2017. https:/doi.org/ 10.1111/cdep.12207

31. PATTERSON, Charlotte. Lesbian and gay parents and their children: a social science perspective, HOPE, Debra (Ed.). Contemporary perspectives on lesbian, gay, and bisexual identities. New York: Springer, 2009. p. 141-182. https://doi. org/10.1007/978-0-387-09556-1 
32. PERERA, Maricela. A propósito de las representaciones sociales: apuntes teóricos, trayectoria y actualidad. Centro de Investigaciones Psicológicas y Sociológicas, CIPS, La Habana 2003.

33. PICHARDO, José Ignacio; DE STÉFANO, Matías; MARTíN-CHIAPPE, María Laura. (Des)naturalización y elección: emergencias en la parentalidad y el parentesco de lesbianas, gays, bisexuales y transexuales. Revista de Dialectología y Tradiciones Populares, v. 70, n. 1, p. 187-203, 2015. https://doi.org/10.3989/ rdtp.2015.01.009

34. PICHARDO, José Ignacio. (Homo)sexualidad y familia: cambios y continuidades al inicio del tercer milenio. Política y Sociedad, v. 46, n. 1-2, p. 143-160, 2009.

35. QUINTERO, Ángela María. Trabajo social y procesos familiares. Buenos Aires: Lumen Humanitas, 1997.

36. STACEY, Judith; BIBLARZ, Timothy. (How) Does the sexual orientation of parents matter? American Sociological Review, v. 66, n. 2, p. 159-183, 2001.

37. VARGAS, Elvira; RIPOLL, Karen; CARRILLO, Sonia; RUEDA, Miguel; CASTRO, John Alexander. Experiencias familiares de madres y padres con orientaciones sexuales diversas. Aportes de la investigación. Bogotá: Ediciones Uniandes, 2011.

38. WESTON, Kath. Las familias que elegimos: lesbianas, gays y parentesco. Barcelona: Ediciones Bellaterra, 2003.

39. WEEKS, Ruth; DERDEYN, Andre; LANGMAN, Margaretha. Two cases of children of homosexuals. Child Psychiatry and Human Development, n. 6, p. 26-32, 1975.

40. ZAMBRANO, Elizabeth. Parentalidades "impensáveis": pais/mães homossexuais, travestis e transexuais, Horizontes Antropológicos, v. 12, n. 26, p. 123-147, 2006.

41. ZAPATA, Bárbara. Las voces de la homoparentalidad. Resultados preliminares de una investigación, Revista de Trabajo Social, n. 15, p. 41-58, 2013.

Recibido: 21 julio 2019.

Aprobado: 15 abril 2020. 
